Surgery for

Congenital Heart

Disease

\title{
Pulmonary atresia with ventricular septal defects and major aortopulmonary collateral arteries: Unifocalization brings no long-term benefits
}

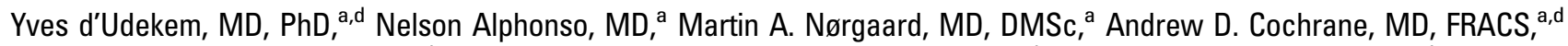
Leeanne E. Grigg, MBBS, FRACP, ${ }^{b}$ James L. Wilkinson, MB, ChB, FRCP, FRACP, ${ }^{c, d}$ and Christian P. Brizard, MD ${ }^{a, d}$

From the Departments of Cardiac Surgery ${ }^{\mathrm{a}}$ and Cardiology, ${ }^{\mathrm{c}}$ Royal Children's Hospital, the Department of Pediatrics, The University of Melbourne, ${ }^{\mathrm{d}}$ and the Department of Cardiology, Royal Melbourne Hospital, ${ }^{\text {b }}$ Melbourne, Australia.

Read at the Eighty-fifth Annual Meeting of The American Association for Thoracic Surgery, San Francisco, Calif, April 10-13, 2005.

Received for publication March 22, 2005; revisions received June 16, 2005; accepted for publication July 15, 2005.

Address for reprints: Yves d'Udekem, MD, $\mathrm{PhD}$, Department of Cardiac Surgery, Royal Children's Hospital, Flemington Rd, Parkville, Melbourne 3052, Victoria, Australia (E-mail: yves.dudekem@rch.org.au).

J Thorac Cardiovasc Surg 2005;130:1496-502

$0022-5223 / \$ 30.00$

Copyright () 2005 by The American Association for Thoracic Surgery

doi:10.1016/j.jtcvs.2005.07.034
Objectives: We sought to evaluate the contribution of unifocalization procedures in the management of patients with pulmonary atresia, ventricular septal defect, and major aortopulmonary collateral arteries.

Methods: From 1975 through 1995, 82 consecutive patients were entered in a multistage approach and had 189 sternotomies and thoracotomies to perform 119 shunts, 130 major aortopulmonary collateral artery transplantations, and 76 major aortopulmonary collateral artery ligations. The serial angiographies and the follow-up of these patients were reviewed.

Results: The concurrent follow-up rate was $80 \%$. The hospital mortality of the preliminary procedures was $4 \%$ (7/189). Fifty-three $(65 \%)$ patients had a complete repair. The hospital mortality of the repair was $8 \%$ (4/53), and 9 late deaths occurred after repair, all of which were cardiac related. The overall survival of all patients to the age of 30 years was $58 \% \pm 7 \%$. Survival 12 years after complete repair was $51 \% \pm$ $14 \%$. On angiography, central shunts promoted growth of central pulmonary arteries in all cases (29 patients). Sixty unifocalized major aortopulmonary collateral arteries were identified in 31 patients. After a mean of $3.2 \pm 4$ years, 26 thrombosed, and 12 presented with a stenosis of greater than $50 \%$. Serial measurements of 29 major aortopulmonary collateral arteries showed no signs of growth $(P=.25)$.

Conclusion: Long-term survival into adulthood of patients with pulmonary atresia, ventricular septal defect, and major aortopulmonary collateral arteries has been achieved with a multistage approach. However, late survival depends exclusively on the growth of the native pulmonary circulation. The few unifocalized major aortopulmonary collateral arteries that did not thrombose failed to grow.

$\mathrm{U}$ ntil recently, the outcomes of patients born with pulmonary atresia, ventricular septal defect (VSD), and major aortopulmonary collateral arteries (MAPCAs) were extremely grim. In the early surgical era, these patients had only a $20 \%$ chance of being alive at the age of 30 years. ${ }^{1}$ Despite numbers of enthusiastic reports from different surgical teams, there is only scarce evidence that surgical management of these patients has resulted in any improvement in their long-term outcomes. The published data are limited to surgical series with maximal follow-up of 10 years. ${ }^{2-4}$ 


$$
\begin{aligned}
& \text { Abbreviations and Acronyms } \\
& \begin{aligned}
\text { LV } & =\text { left ventricular } \\
\text { MAPCA } & =\text { major aortopulmonary collateral artery } \\
\text { PA } & =\text { pulmonary artery } \\
\text { RV } & =\text { right ventricular } \\
\text { TOF } & =\text { tetralogy of Fallot } \\
\text { VSD } & =\text { ventricular septal defect }
\end{aligned}
\end{aligned}
$$

In the early 1980s, a policy of multistage operations to achieve a complete repair of these lesions was initiated in Melbourne by Dr R. B. Mee and later followed by Drs W. J. Brawn and T. R. Karl. ${ }^{5}$ These operations consisted of preparatory procedures consisting of central shunting and unifocalization of MAPCAs with native pulmonary artery (PA) branches performed by multiple sternotomies and thoracotomies. Once a reasonable pulmonary vascularization had been obtained in these patients, they were offered a complete repair with transventricular closure of the VSD and implantation of a valved conduit between the right ventricle and the PA. We reviewed the long-term outcome of the patients who underwent this multistage approach.

\section{Patients and Methods}

The design of the study was submitted and approved by the hospital ethics committee. The data of all patients with pulmonary atresia, VSD, and MAPCAs who took part in the policy of staged repair in the Royal Children's Hospital, Melbourne, between January 1979 and May 1995 were reviewed. To evaluate the fate of the patients born with MAPCA-dependent lung circulation, we excluded those patients with ductus-dependent circulation and those with tetralogy of Fallot who had PAs of reasonable size.

Eighty-four patients were offered surgical intervention. Two patients who had exploratory median sternotomies during which no central PA could be found were offered no further treatment. The remaining 82 patients constitute the core of this study. The characteristics of the patients before the first operation are given in Table 1 .

The patients underwent 67 sternotomies, 61 right thoracotomies, and 61 left thoracotomies to perform the preparatory procedures described in Table 2. The median age at the time of the first operation was 1.4 years (range, 7 days to 34 years). Nineteen patients had only one procedure, and the remaining had a mean of $2.6 \pm 0.8$ procedures during a median time interval of 1.6 years (range, same day to 10 years later).

The last follow-up of the patients was obtained from their referring cardiologists. Angiograms were reviewed several times to identify the effect of the different procedures performed on PA growth. Because of the diversity of the operations, attention was focused on the results of 3 standard procedures: central shunt, MAPCA transplantation, and PA patch enlargement. When the left PA or a transplanted MAPCA could be identified on serial examinations, their caliber and the size of a 1-cm mark displayed on the grid filmed at the time of the angiogram were measured. Care was taken to measure each MAPCA of a given patient at a similar level
TABLE 1. Patient characteristics

\begin{tabular}{lc}
\hline No. of patients & $\mathbf{8 2}$ \\
\hline Sex (male/female) & $51 / 31$ \\
Symptoms & \\
Asymptomatic & 4 \\
Cyanosis & 48 \\
Heart failure & 10 \\
Cyanosis + heart failure & 18 \\
Unknown & 3 \\
Mean arterial saturation* & $81 \% \pm 9 \%$ \\
Patients with no confluent PAs* & 9 \\
Mean no. of MAPCAs per patient & $4 \pm 1.4$ \\
\hline
\end{tabular}

$\overline{P A}$, Pulmonary artery; MAPCAs, major aortopulmonary collateral arteries. *Data obtained during catheterization.

devoid of focal stenosis. The absolute size of the vessels was then calculated by dividing the measurement of the vessel by the measurement of the 1-cm mark.

Survival analysis was performed by the Kaplan-Meier method for the whole patient group and separately for those patients who reached full repair and those who received "palliative surgery." Survival curves were compared by the log-rank test.

The measured PA and MAPCA diameters were compared with paired $t$ tests. An analysis of prognostic or risk factors for survival or death was performed by logistic regression, with initial univariate and subsequent multivariate analysis. A Cox proportional hazards analysis was performed for the same prognostic factors and survival time. These 2 analyses were performed for the total patient group $(\mathrm{n}=82)$ and also for those who reached full repair $(\mathrm{n}=53)$.

The prognostic factors included in the analysis were sex, symptomatic status, age at the time of the first operation, arterial oxygen

\section{TABLE 2. Preparatory procedures}

\begin{tabular}{lr}
\hline Procedures & No. \\
\hline Central shunt & \\
MPA implantation & 35 \\
Polytetrafluoroethylene conduit & 16 \\
RVOT patch & 5 \\
RV-to-PA conduit & 7 \\
Right modified BT shunt & 17 \\
Left modified BT shunt & 14 \\
Shunt from aorta to PA & 9 \\
PA patch enlargement & 13 \\
Shunt to MAPCAs & 16 \\
MAPCAs transplantation & 130 \\
MAPCAs ligation & 76 \\
Azygos vein interposition & 9 \\
Pericardial roll PA creation & 9 \\
Mitral valve repair & 1 \\
Right upper lobe bronchus resection & 1 \\
\hline
\end{tabular}

$M P A$, Main pulmonary artery; RVOT, right ventricular outflow tract; $R V$, right ventricle; $P A$, pulmonary artery; $B T$ shunt, Blalock-Taussig shunt; $M A P C A s$, major aortopulmonary collateral arteries. 


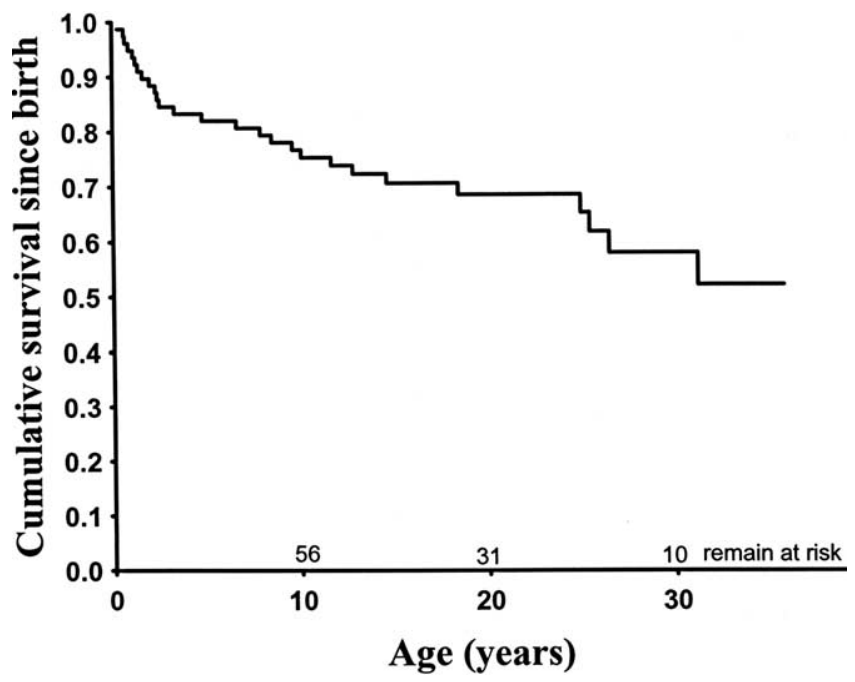

Figure 1. Kaplan-Meier curve of survival since birth of all patients.

saturation, the presence of confluent PAs, the number of MAPCAs, the performance of a central shunt or a peripheral shunt, age at full repair, total number of operations, right ventricular (RV) systolic pressure at late follow-up, and PA pressure at follow-up.

The analyses were performed with Stata version 8 software (Stata Corp, College Station, Tex).

\section{Results}

\section{Procedural Outcome}

The hospital mortality of the preparatory procedures was $4 \%$ (7 patients). Two patients died of pulmonary hemorrhage, and 1 died of bleeding related to the rupture of a suture line of a conduit between the right ventricle and the PA. Four patients aged $2,12,14$, and 17 months, respectively, died of the consequences of hypoxia after either central shunts or unifocalizations. One patient went into heart failure after unifocalization and underwent a successful early repair 3 days after that procedure. The condition of 22 patients was deemed unrepairable after the last palliative procedure.

Complete repair was achieved in 53 patients at a median of 9 months (range, same day to 5 years later) after the last preparatory procedure. The median age at the time of the repair was 4 years (range, 3 months to 35 years). The repair consisted of a transventricular patch closure of the VSD and the placement of a conduit between the ventriculotomy and the main PA. The conduits used were homografts $(n=23)$, bioprosthetic valved conduits $(n=28)$, and mechanical valves with patches $(n=2)$. Additionally, 5 MAPCAs were transplanted and 2 were ligated, and 6 right and 1 left PAs underwent patch enlargement. Four patients had patch enlargement of both PAs, and 1 patient underwent a reconstruction of both central PAs with a pericardial roll. One patient was left with a fenestrated VSD, which was subse-

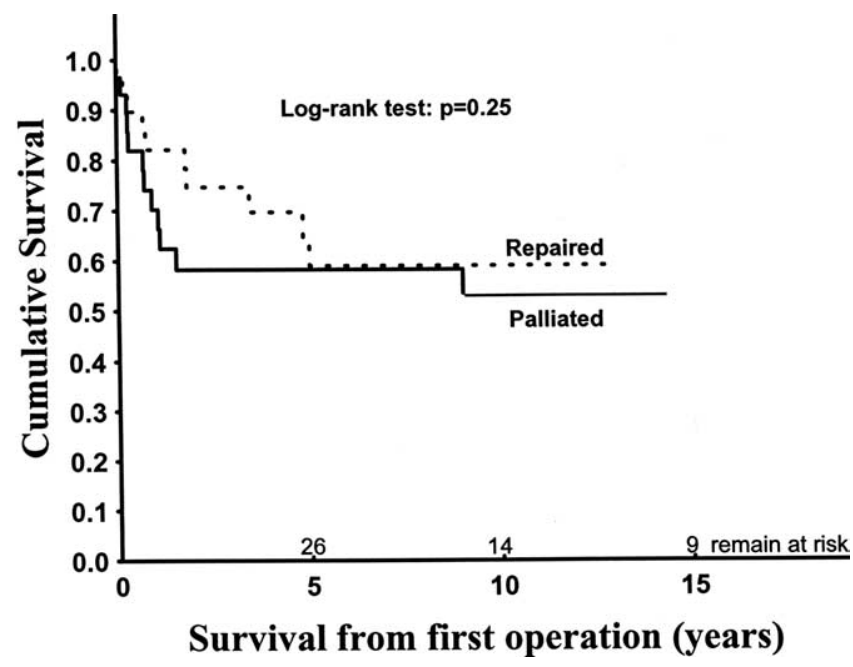

Figure 2. Kaplan-Meier curves of survival since first procedure.

quently closed during the same hospital stay. The hospital mortality of the repair was $8 \%$ (4 patients). Two infants died of respiratory failure related to severe bronchomalacia. One patient who maintained suprasystemic RV pressures died during a pulmonary hypertensive crisis, and the last patient died from uncontrollable bleeding from the right upper lobe bronchus.

\section{Follow-up}

The concurrent follow-up rate (2003-2004) was $80 \%$. The median follow-up time was 14.2 years (range, 3 months to 25 years), for a total of 967 patient-years. Seven of the 22 patients whose condition was believed to be unrepairable died a median of 8 years (range, 3 months to 13 years) after the last procedure. The cause of death was hemoptysis $(\mathrm{n}=$ $2)$, hypoxia $(\mathrm{n}=1)$, endocarditis $(\mathrm{n}=1)$, pulmonary hemorrhage related to the rupture of PA during an interventional catheterization procedure $(\mathrm{n}=1)$, and unknown $(\mathrm{n}=$ 2 ). One patient was lost to follow-up. One patient underwent heart-lung transplantation 9 years after the last palliative procedure and was still alive 14 years after the transplantation. The remaining palliated patients were still alive after a median of 13 years (range, 9-25 years). At last follow-up, 1 patient was in New York Heart Association class I, 8 were in class II, and 4 were in class III.

Two of the patients who underwent a complete repair were lost to follow-up. Nine late deaths occurred a median of 4 years (range, 4 months to 21 years) after repair. Four patients died of RV failure, 2 patients had a sudden death (one of whom was known to have pulmonary hypertension), and 2 patients died after reoperations ( 1 for tracheal stenosis and 1 for homograft replacement). The cause of death could not be determined in 1 patient. The clinical status of 1 living 


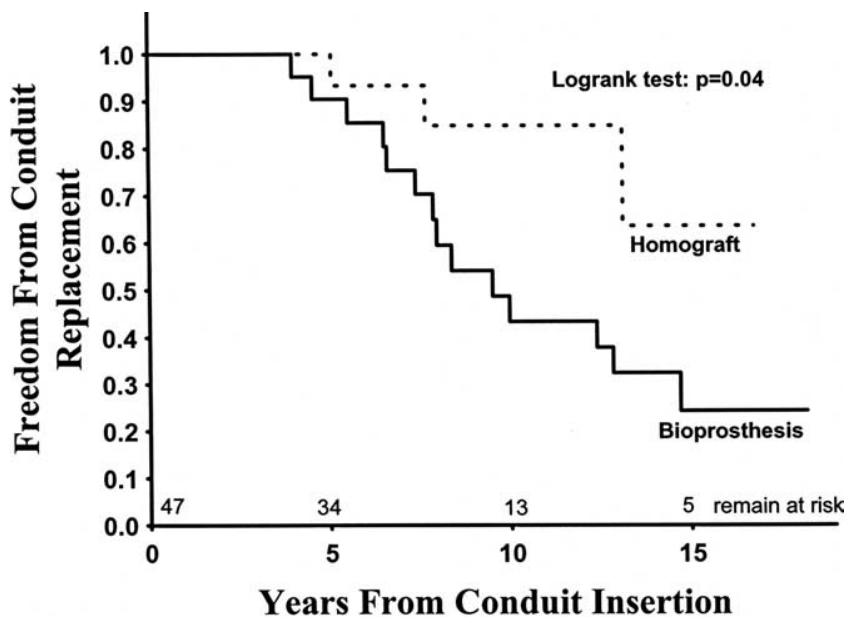

Figure 3. Freedom from reoperation for right ventricle-to-pulmonary artery conduit replacement.

patient could not be ascertained. Twenty-five (66\%) of the remaining 38 patients were in New York Heart Association class I, 11 (29\%) were in class II, and 1 was in class III. Sixty-nine percent $(9 / 13)$ of the palliated patients and $96 \%$ (29/30) of the repaired patients worked or studied. None of the palliated patients practiced sport, whereas $57 \%(16 / 28)$ of the repaired ones engaged in regular sporting activities.

The Kaplan-Meier curve of survival since birth of the whole group of patients, irrespective of whether a repair could be performed, is displayed in Figure 1. The estimated probability to reach the age of 30 years was $58 \% \pm 7 \%$. Kaplan-Meier curves of survival after the first operation were not significantly different for repaired patients and for those who remained palliated $(P=.25$, log-rank test; Figure 2 ). After 12 years, the survival of the patients who under- went a complete repair was $51 \% \pm 13 \%$. Their chances of being alive and in class I at 12 years was $32 \% \pm 10 \%$.

Cox proportional hazards analysis demonstrated that older age at the time of the first operation taken as a continuous variable was the only significant but weak factor predictive of longer late survival for each additional month of age at the time of the operation $(P=.02$, hazard ratio $=0.99)$.

Eighteen patients had a total of 22 redo procedures for replacement of the conduit between the right ventricle and the PA. The first replacement of the conduit was performed a median of 8 years (range, 4-15 years) after repair. The freedom from reoperation for conduit replacement is displayed in Figure 3. Ten years after repair, the freedom from reoperation was higher for patients who had a homograft implanted than for those with a bioprosthetic conduit $(85 \% \pm$ $10 \%$ vs $43 \% \pm 11 \% ; P=.04, \log$-rank test).

\section{Angiographic Studies}

A total of 268 angiograms were reviewed. The effects of the central shunt could be evaluated in 29 patients. Twentythree had their main PA directly implanted in the ascending aorta, and 6 had polytetrafluoroethylene shunts (Gore-Tex shunt; W. L. Gore \& Associates, Inc, Flagstaff, Ariz). One polytetrafluoroethylene central shunt blocked and all others promoted growth of the central PAs. Eleven (39\%) right and $2(7 \%)$ left PAs showed stenosis of greater than $50 \%$. All of the PA stenoses occurred in the patients who had a main pulmonary implantation in the aorta. The left PA diameter increased from $3 \pm 1$ to $6 \pm 2 \mathrm{~mm}(P<.001)$ in the 2 -year interval after shunt completion.

Only 60 unifocalized MAPCAs could be identified on serial angiograms in 31 patients, an example of which is given in Figure 4. After a mean of $3.2 \pm 4$ years, 26 had thrombosed, and 12 presented with stenosis of greater than $50 \%$. Serial measurements of 29 MAPCAs were obtained.
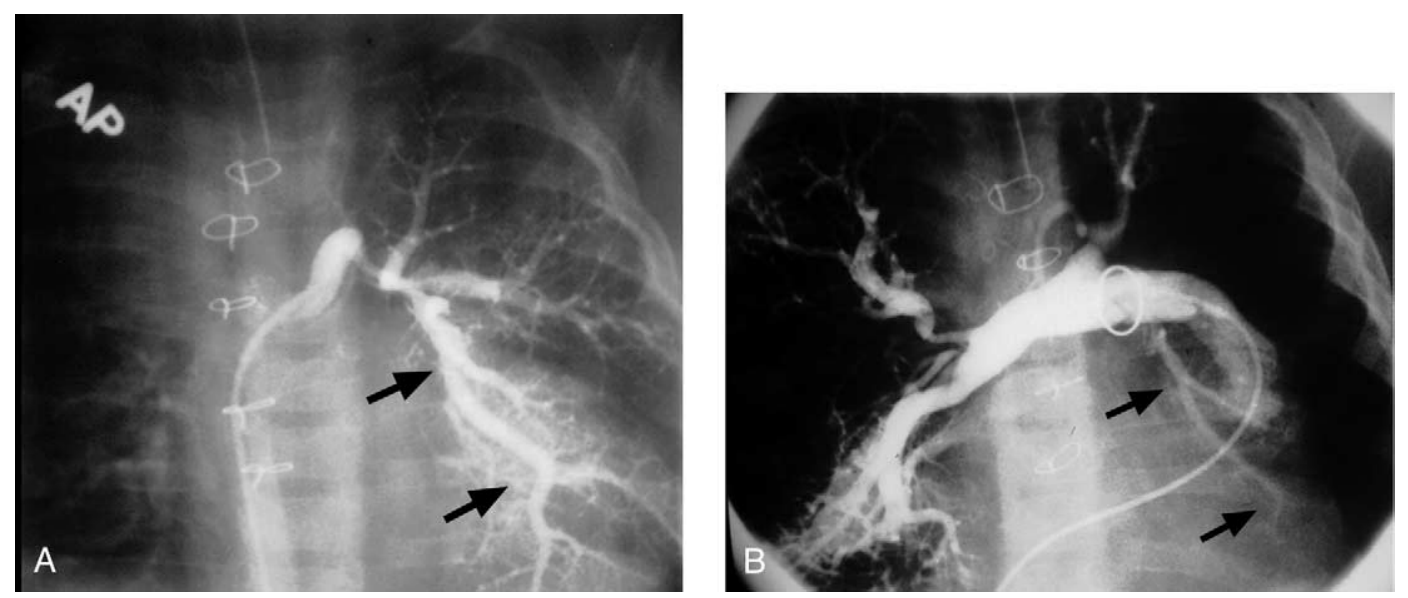

Figure 4. Left major aortopulmonary collateral artery before unifocalization (A) and 4 years after unifocalization (B). 
Over a mean time interval of 3.5 years, during which the weight of the patients increased from $12 \pm 9$ to $25 \pm 21 \mathrm{~kg}$ $(P<.001)$, the MAPCA size went from $3.6 \pm 1$ to $4 \pm 2$ $\mathrm{mm}(P=.25)$.

PA patch enlargement could be assessed angiographically for 21 patched arteries in 14 patients. After a median of 2.9 years (range, 8 months to 13 years), 4 patched arteries showed stenosis of greater than $50 \%$. Three were successfully dilated, and the fourth patient died of bleeding subsequent to the balloon dilatation of the PA.

We collected invasive measurements of RV pressures obtained during angiography in patients who had undergone a successful repair. Data were available in 22 patients who had no RV outflow tract obstruction a median of 1.38 years (range, 7 days to 9 years) after the repair. The mean ratio between systolic right and left ventricular pressures was $0.62 \pm 0.32$. Four patients had suprasystemic RV pressures. Two of these patients died in the month after this angiogram.

\section{Discussion}

Our study set a landmark for the expectations of patients with pulmonary atresia, VSD, and MAPCA-dependent lung circulation when offered a multistage approach of multiple operations aiming toward the completion of a complete repair. This 20-year work achieved by our predecessors in Melbourne shows a remarkable improvement in survival and quality of life among children affected by this condition. There are not many data concerning survival into adulthood for these patients. Bull and associates, ${ }^{1}$ in 1995, predicted a $20 \%$ chance of survival to the age of 30 years. These results were so disappointing that they wondered whether surgical intervention did not adversely affect the outcome of the patients. With a multistage approach, the chance of survival to the age of 30 years increased to $60 \%$. Importantly, repair was possible in $65 \%$ of the patients. There seems to be attrition in the number of survivors in the first few years after repair because of right heart failure in patients with poorly developed pulmonary vascular bed and high pulmonary pressures. Nonetheless, we have hope that survivors of these first years after repair will now have long-lasting results. All of them now lead active normal lives. Surgical practice is constantly evolving, and the actual results should be the standards to which further changes in practice should be compared.

There has not yet been a consensus on the long-term fate of the MAPCAs. A study from the Great Ormond Street Hospital in 1984 raised the suspicion that growth could not be obtained from MAPCAs. ${ }^{6}$ The study was based on procedures performed in an early surgical era, and a lot of the connections between MAPCAs and native PAs were made with interposition of prosthetic material. This study did not dampen the enthusiasm of surgeons to integrate MAPCAs in a newly reconstructed pulmonary circulation, with various approaches, techniques, and timing. ${ }^{7-10}$ Most of the MAPCA transplantations described in our study were performed through thoracotomies, which seems technically easier than performing them through sternotomies, by surgeons who have proved their technical excellence. Despite this ideal setting, the results of unifocalization were disappointing. These are technically demanding operations, and this explains the high rate of thrombosis of these vessels. But even when the collateral vessels remained patent, they did not exhibit any growth. Therefore we believe that, at the time of their transplantation, collateral vessels have already acquired their maximum development and that their integration in the pulmonary circulation will not bring any long-term benefit to the patient because they do not seem to contribute to the definitive lung circulation of the repaired patients.

On the other hand, the shunting procedures have been extremely efficient in developing even diminutive native pulmonary circulation, and the long-term survival of the patients and their quality of life seemed to rely mainly on the growth of the native vessels by these shunting procedures. A high incidence of right PA stenosis was observed after reimplantation of the main PA in the ascending aorta. After this procedure, the right PA, tightly applied against the back of the aorta, might become compressed.

In such a long-term retrospective study, there are natural limitations. Patients who presented for surgical intervention were older than those presenting nowadays. Some of them might represent survivors of a natural selection process.

One could also argue that vessels transplanted sooner after birth might have better growth potential. However, our results showed so consistently any lack of growth of the MAPCAs, that it is difficult for us to believe that an earlier procedure might result in a potential advantage, especially given that shunting procedures performed during the same time frame predisposed to the growth of the native vessels.

On angiography, the identification of a MAPCA is eased by the recognition of its peculiar morphology, and the longer the tract of the transplanted MAPCA before it reaches the lung, the easier its identification. Therefore it is possible that some MAPCAs transplanted very distally at the surface of the lung might have a better outcome and could not be identified.

Finally, it is likely that the patients who were investigated by means of angiography early after repair were those who were symptomatic. The reported PA pressures were therefore possibly overestimated.

In conclusion, long-term survival into adulthood of patients with pulmonary atresia, VSD, and MAPCAs has been achieved with a multistage approach. In our series, unifocalized MAPCAs exhibited a high incidence of occlusion and no growth. Therefore in patients with pulmonary 
atresia, VSD, and MAPCAs, operations should aim at achieving growth of the native PAs by shunting rather than unifocalization.

\section{References}

1. Bull K, Somerville J, Ty E, Spiegelhalter D. Presentation and attrition in complex pulmonary atresia. J Am Coll Cardiol. 1995;25:491-9.

2. Griselli M, McGuirk SP, Winlaw DS, Stumper O, de Giovanni JV, Miller P, et al. The influence of pulmonary artery morphology on the results of operations for major aortopulmonary collateral arteries and complex congenital heart defects. J Thorac Cardiovasc Surg. 2004; 127:251-8.

3. Reddy VM, McElhinney DB, Amin Z, Moore P, Parry AJ, Teitel DF, et al. Early and intermediate outcomes after repair of pulmonary atresia with ventricular septal defect and major aortopulmonary collateral arteries: experience with 85 patients. Circulation. 2000;101: 1826-32.

4. Gupta A, Odim J, Levi D, Chang RK, Laks H. Staged repair of pulmonary atresia with ventricular septal defect and major aortopulmonary collateral arteries: experience with 104 patients. $J$ Thorac Cardiovasc Surg. 2003;126:1746-52.

5. Iyer KS, Mee RB. Staged repair of pulmonary atresia with ventricular septal defect and major systemic to pulmonary artery collaterals. Ann Thorac Surg. 1991;51:65-72.

6. Sullivan ID, Wren C, Stark J, de Leval MR, Macartney FJ, Deanfield JE. Surgical unifocalization in pulmonary atresia and ventricular septal defect. A realistic goal? Circulation. 1988;78(suppl III):III5-13.

7. Sawatari K, Imai Y, Kurosawa H, Isomatsu Y, Momma K. Staged operation for pulmonary atresia and ventricular septal defect with major aortopulmonary collateral arteries. New technique for complete unifocalization. J Thorac Cardiovasc Surg. 1989;98:738-50.

8. Reddy VM, Liddicoat JR, Hanley FL. Midline one-stage complete unifocalization and repair of pulmonary atresia with ventricular septal defect and major aortopulmonary collaterals. J Thorac Cardiovasc Surg. 1995;109:832-44.

9. Yagihara T, Yamamoto F, Nishigaki K, Matsuki O, Uemura H, Isizaka $\mathrm{T}$, et al. Unifocalization for pulmonary atresia with ventricular septal defect and major aortopulmonary collateral arteries. J Thorac Cardiovasc Surg. 1996;112:392-402.

10. Luciani GB, Wells WJ, Khong A, Starnes VA. The clamshell incision for bilateral pulmonary artery reconstruction in tetralogy of Fallot with pulmonary atresia. J Thorac Cardiovasc Surg. 1997;113:443-52.

\section{Discussion}

Dr Frank L. Hanley (Stanford, Calif). This is an outstanding article, and I have absolutely no quarrels with your presentation, data, or research methods. I do have a major difference, however, with the inferences that you have drawn and where you want to go in the future.

My comments are designed to explain my reasons for disagreeing with your proposed management scheme of this lesion.

Tetralogy of Fallot (TOF)/PA/MAPCAs manifests a wide spectrum of pulmonary vascular morphology and physiology, ranging from patients on the favorable end with normally arborizing true PAs with the collaterals simply contributing systemic flow into the PA system to patients on the unfavorable end with completely absent true PAs and all of the pulmonary blood supply from collaterals. Most patients fall in the continuum between these 2 extremes, with PAs present but having significant arborization abnormalities and important segments of lung solely supplied by collaterals. Any lesion with this degree of variability requires a carefully formulated management protocol on the basis of morphology and physiology.
TOF/PA/MAPCAs, in particular, is in great need of such a protocol. The goal of any protocol is to achieve full repair with the lowest possible PA pressure in the greatest number of patients. Too often the recommendation is made simply to place a shunt in cyanotic patients or to observe growing infants who have adequate systemic oxygen saturation. These approaches, which are tried and true for other cyanotic patients who have a single PA compartment and a single source of pulmonary blood flow, can be disastrous in a patient with a multicompartmentalized PA vascular system that yields widely varying pressures and flows in different lung segments. Contrary to the typical cyanotic patient, a patient with MAPCAs well balanced on the outside is not necessarily well balanced on the inside.

The authors are to be congratulated for recognizing the need for an organized management protocol for this patient population and for formulating and then proposing such a protocol. They have presented valuable and credible late follow-up information on a relatively large series of patients and have done so in a clear and organized manner. The experience demonstrates a clear improvement on the natural history of this disease and represents an important historical chapter in the evolution of the thinking as it relates to the management of TOF/PA/MAPCAS. I respectfully but strongly disagree, however, with the inferences that the authors have drawn and thus with the details of their proposed management protocol for future patients. The authors have concluded that surgically bringing collaterals into the PA vascular system has no benefit. Their reasoning is that unifocalized collaterals commonly thrombose, and if they stay patent, they do not grow. Thus they recommend stimulating growth of the true PAs, regardless of the arborization profile of the PAs or the collateral distribution, using a surgically placed central shunt. After the PAs enlarge, patients are evaluated for VSD closure and RV outflow tract conduit placement. Unifocalization is not included as a central component of the management plan.

This approach runs counter to all of the principles for promoting a complete and healthy pulmonary vascular bed, which is the most important criterion in achieving the stated goal of full repair with low PA pressure. Untouched collateral vessels are exposed to systemic level pressure and high flow from birth, and they, as well as the distal lung, begin to degenerate on a time course measured in weeks to months. By 6 months of age, some lung segments will be lost to collateral occlusion, and others will be at risk of pulmonary microvascular disease. The result is a limited peripheral vascular bed that is unhealthy. The approach recommended by the authors can work well but only in a limited band of patients: those with a favorable true PA arborization profile. The remainder will suffer from this approach. This observation is supported by the outcome data. Fifty-eight percent (49/84) of patients in the authors' series survived with full repair, and the mean $\mathrm{RV} / \mathrm{left}$ ventricular $(\mathrm{LV})$ pressure ratio was 0.62 . This mean pressure value is on the border of what is considered unacceptable hemodynamics $(\mathrm{RV} / \mathrm{LV}$ ratio $>0.67)$, and therefore about half of the patients repaired had an unacceptable result. Thus about $30 \%$ of all patients achieved a hemodynamically acceptable full repair. The median age at repair was 4 years, and it required 2.6 operations to achieve full repair.

We also have developed a management protocol for this lesion, but it differs substantially from that of the authors. Our approach 
involves evaluating all patients in the newborn period in anticipation of achieving unifocalization of all collaterals by the age of 3 to 4 months. The overriding concern is to get all collaterals out of the high-pressure and flow systemic circulation as soon as possible and create a 1-compartment pulmonary vascular system. Specific morphology and physiology dictate the exact approach. In more than half of all patients, a 1-stage complete unifocalization and intracardiac repair is performed at 3 to 4 months. In the majority of the remainder, complete unifocalization is performed, but the intracardiac repair is deferred until a later time. In a small group of patients, about $15 \%$, a central aortopulmonary window or a lateral thoracotomy is performed. Our personal series of 289 total patients reveals a total of 1069 collaterals. Essentially all collaterals were unifocalized. Early mortality occurred in $5.1 \%$ of patients with TOF/PA/MAPCAs managed by us from the beginning. In the typical patient, if we can call it that, the true PAs communicated with less than half of the lung parenchyma. Our data strongly indicate that collaterals, when managed appropriately with respect to timing and technique, do grow and grow well. To emphasize this point, our subgroup of patients with completely absent true PAs were reconstructed with only collateral tissue, and this subgroup has done just as well as the larger group over the long term. I cite the example of a patient with 4 collaterals and no PAs who underwent a 1-stage complete unifocalization and intracardiac repair at 5 months of age. The early postoperative $\mathrm{RV} / \mathrm{LV}$ ratio was 0.45 . This patient returned for conduit change at age $71 / 2 \mathrm{yrs}$. The collateral tissue that solely made up his pulmonary vasculature had developed in parallel with his somatic growth, and in fact, his $\mathrm{RV} / \mathrm{LV}$ ratio was now 0.32 . An earlier analysis of our experience showed that after unifocalization, collateral occlusion is unusual, if not rare, but about $32 \%$ of patients will need an intervention on the peripheral pulmonary vasculature at 2-year follow up, about the equivalent follow-up time cited by the authors for their evaluation of collateral patency.

Most importantly, after this management protocol, a full repair rate of $83 \%$ was achieved. The mean $\mathrm{RV} / \mathrm{LV}$ ratio was 0.43 (SD \pm 0.13 ), confirming that the large majority of full repairs were accompanied by acceptable hemodynamics (ie, an RV/LV ratio $<$ $0.67)$. Thus about $80 \%$ of all cases received a hemodynamically acceptable full repair at a mean age of 0.6 years for the complete repairs. In $68 \%$ of full repairs, it was accomplished in 1 operation.

The experience described by the authors is an important and impressive achievement, but it must be placed in the historical context of the evolution of thinking relating to this disease process and not used as a basis for formulating new and improved protocols. Current thinking, and the data to back up that thinking, strongly support moving in a direction opposite that recommended by the authors. The protocol recommended by the authors going forward in essence sets the bar too low. It is effective for a very limited subgroup of patients with TOF/PA/MAPCAs but is not designed to achieve the most good for the greatest number of patients.

Therefore, again, I have no quarrel with your data. Mostly, I would like to understand a little bit better your thinking on why your proposed management plan is good for the majority of patients, who will be left with a limited pulmonary vascular bed after taking this approach.

Dr d'Udekem. Thank you very much. I have to disagree along several points.

First, I believe that since we have adopted this approach of shunting all neonates in the first few weeks of life, performing repeatedly right ventricle-to-PA conduits and abandoning unifocalizations, we achieved excellent outcomes, not for part of the patients but for almost all of them. And we have been very successful thus far by doing just simple procedures.

The next point concerns what you said about the goal to achieve, being a repair with the lowest possible PA pressures. Anybody in the audience, I believe, has mortality for this condition that varies between $10 \%$ and $20 \%$ at best. I think that the goal for these patients is to reduce this mortality to the maximum. We should have the lowest possible mortality. The London team showed it 10 years ago, and it is still the same with this historical series. Procedures and operations kill patients more surely than their natural history. If we manage to have operations with no mortality, we will improve the patient's outcome because we will improve their survival. I think it is better than achieving repair at all cost. We had 6 deaths after repair because of pulmonary hypertension. It is better to have a patient who is alive at 30 or 40 years of age with $85 \%$ saturation than dying 2 years after a repair with $98 \%$ saturation.

The next point is the assumption that we will select patients with a favorable anatomy. I think one of the major mistakes that has been done in the past, and that is what we believe, is that we are waiting too long to operate on these patients. And that is a point of view that you defended in the past. I do not know the numbers that you quoted today. I know only your publication from 2000, in which the median age at the first operation was 7 months. And that was the earliest median age at first operation that appeared in the literature.

I think that you have to attack these native PAs very early after birth. The reason why you get these unfavorable anatomies is because you wait too long. I have reviewed all the angiograms from this series. All the patients who were operated on before 1 month of age had central PAs. And since we have adopted this new approach, we did not have patients with no central PAs. We always have something to put a shunt on.

Dr Williams. Yves, I think in fairness to the final article, we will interrupt your discussion. We obviously have 2 very strong points of view, and we will let you sort it out. 